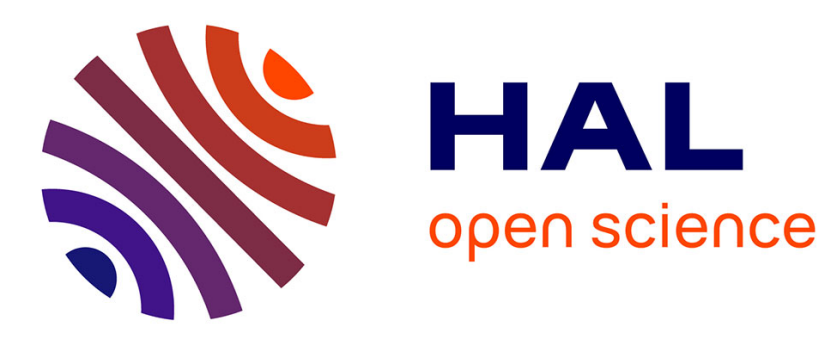

\title{
Caractérisation mécanique des solides par spectro-interférométrie ultrasonore
}

J. Roux, B. Hosten, Bernard Castagnede, M. Deschamps

\section{To cite this version:}

J. Roux, B. Hosten, Bernard Castagnede, M. Deschamps. Caractérisation mécanique des solides par spectro-interférométrie ultrasonore. Revue de Physique Appliquée, 1985, 20 (6), pp.351-358. 10.1051/rphysap:01985002006035100 . jpa-00245343

\section{HAL Id: jpa-00245343 https://hal.science/jpa-00245343}

Submitted on 1 Jan 1985

HAL is a multi-disciplinary open access archive for the deposit and dissemination of scientific research documents, whether they are published or not. The documents may come from teaching and research institutions in France or abroad, or from public or private research centers.
L'archive ouverte pluridisciplinaire HAL, est destinée au dépôt et à la diffusion de documents scientifiques de niveau recherche, publiés ou non, émanant des établissements d'enseignement et de recherche français ou étrangers, des laboratoires publics ou privés. 


\title{
Caractérisation mécanique des solides par spectro-interférométrie ultrasonore
}

\author{
J. Roux, B. Hosten, B. Castagnede et M. Deschamps \\ Laboratoire de Mécanique Physique (*), Université de Bordeaux I, 351 cours de la Libération, \\ 33405 Talence Cedex, France
}

(Reçu le 29 novembre 1984, accepté le 4 février 1985)

\begin{abstract}
Résumé. - La caractérisation structurale et mécanique de solides anisotropes naturels tels que le bois, les roches ou les composites artificiels est abordée par spectro-interférométrie ultrasonore dans la bande moyenne 0,5-25 $\mathrm{MHz}$. On montre comment l'acquisition des signaux impulsionnels assistée par ordinateur accroît les performances d'un dispositif à immersion par des techniques de filtrage-corrélation et poursuite de modes.

Des algorithmes d'optimisation des constantes élastiques complexes associés à des jauges d'anisotropie permettent la validation de modèles de comportement mécanique, la mesure des constantes correspondantes et la fiabilité des résultats obtenus.
\end{abstract}

\begin{abstract}
Texture characterization and mechanical behaviour of anisotropic solids as wood, rocks or synthetic composite materials are performed by ultrasonic spectro-interferometry in the middle range of 0.5 to $25 \mathrm{MHz}$. Data handling of pulsed waves fully computer-assisted improves accuracy of an immersion device by filtering correlation techniques and normal modes tracking. Complex elastic constants are optimized from special algorithms associated with anisotropy gauges leading to behaviour laws assessment, including reliability and accuracy of mechanical data.
\end{abstract}

\section{Introduction.}

La métrologie des propriétés élastiques des solides a donné lieu, depuis de nombreuses années à une très grande quantité de travaux. L'idée de base est commune à tous les dispositifs qui consiste à : générer une onde élastique de structure connue; déterminer sa vitesse de propagation et, par l'équation d'onde correspondante, calculer les coefficients mécaniques utiles.

La détermination des vitesses est en général assez facile et très précise soit en résonance, soit en impulsions, pour les matériaux isotropes et faiblement dispersifs. La détermination des amortissements anisotropes est déjà beaucoup moins répandue.

La modélisation mécanique de matériaux naturels (bois, roches) ou de matériaux synthétiques (composites) nécessite en outre une bonne connaissance des vitesses et atténuations sur un spectre de fréquences le plus large possible pour confirmer ou infirmer une loi de comportement.

On trouvera dans les références [1-9] les versions de

(*) U.A., C.N.R.S. 867. base des méthodes à contact direct, en [10] et [11] les méthodes à immersion.

Ce sont ces deux dernières qui sont à l'origine de nos travaux, car la transmission ultrasonore au travers d'une lame à faces parallèles immergée dans un liquide offre un éventail d'applications très large.

D'abord le nombre réduit de coupes orientées pour l'obtention du modèle d'anisotropie à partir des courbes de lenteurs par des mesures à incidence variable. En effet, certains matériaux synthétiques comme les composites à fibres et matrices métalliques ne sont produits qu'en plaques minces; une seule orientation est donc disponible et l'on doit générer au moins deux modes propres dans chaque plan principal pour accéder aux cinq constantes élastiques dans le cas d'un uniaxe. Et ensuite, la reproductibilité de l'interface solide-liquide se prête mieux aux mesures d'amplitude et d'atténuation que les méthodes à contact direct, quel que soit le soin apporté au couplage transducteurmatériau. La contrepartie existe qui est essentiellement la faible transmission par la rupture d'impédance solide-liquide et la réponse impulsionnelle multimodale accompagnant les diverses incidences.

La précision des résultats en est évidemment affectée et les dispositifs de ce type n'offraient pas de perfor- 
mances suffisantes pour la mise en évidence d'anisotropies et de biréfringences induites par contraintes extérieures ou par endommagement, voire pour l'accession aux coefficients élastiques d'ordre supérieur.

\section{Spectro-interférométrie assistée par ordinateur.}

Un interféromètre classique à immersion est représenté sur la figure 1.

La lame à faces parallèles du matériau à tester est mise en incidence variable dans la branche signal entre émetteur et récepteur fixes. La branche référence est constituée d'une paire de transducteurs identiques, dont on peut faire varier la distance avec précision, constituant ainsi une ligne à retard analogique pratiquement sans dispersion. L'émission impulsionnelle assure la séparation temporelle des modes générés par réfraction. La mesure précise des temps de parcours s'obtient par un détecteur de coïncidence entre les signaux issus de la ligne de mesure et de la ligne de référence.

De très nombreux raffinements ont été imaginés pour accroître la résolution du détecteur de coïncidence tant sur les enveloppes des signaux détectés, pour obtenir la vitesse de groupe, que par les détections de phase pour l'obtention de la vitesse de phase.

On peut remarquer dans l'éventail de tous ces dispositifs [1] à [9] leur spécificité métrologique qui conduit à l'abandon d'une grande partie de l'information présente dans les signaux au profit du seul paramètre privilégié. Il est flagrant que les systèmes privilégiant la mesure des vitesses sont inaptes à la détermination des amortissements et réciproquement. L'exploration d'un domaine fréquentiel assez large est souvent difficile sans perte de précision.

Le système que nous avons peu à peu mis au point reprend les fonctions essentielles de la version de base représentée en figure 1. Dans la bande moyenne 0,5-25 $\mathrm{MHz}$, la numérisation des signaux et l'assis-

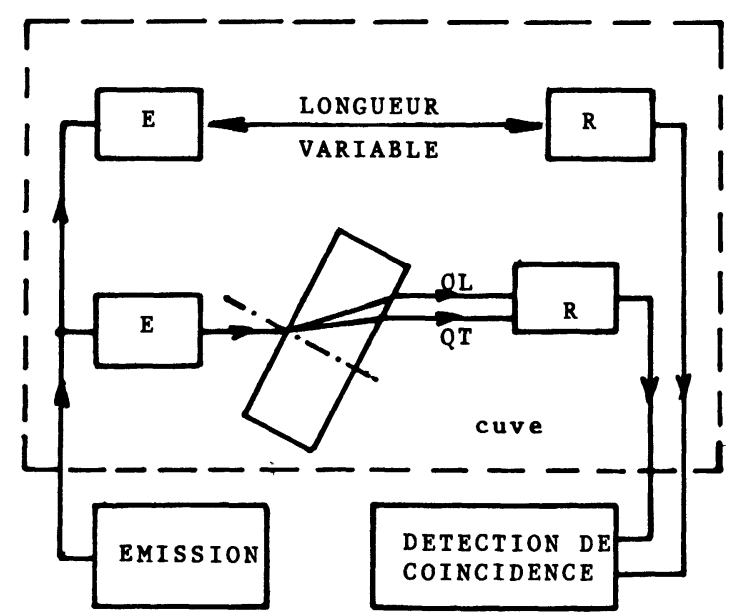

Fig. 1. - Interféromètre ultrasonore à immersion. Schéma de base.

[Basic device for immersion technique.] tance des paramètres d'acquisition par un microordinateur permettent de conserver et d'exploiter la totalité des informations contenues dans les signaux.

2. 1 DisPositif EXPÉRIMENTAL. - La version actuelle est représentée sur le synoptique de la figure 2 .

La cuve à eau est régulée en température par un double circuit thermostaté, la surface libre étant isolée par un couvercle flottant en polystyrène expansé. La stabilité en température obtenue autour de l'ambiante $\left(20^{\circ} \mathrm{C}\right)$ est meilleure que $1 / 100^{\circ} \mathrm{C}$. En effet, un calcul d'incertitude élémentaire montre qu'une résolution de \pm 1 ns dans les temps de parcours nécessite une stabilité du chemin acoustique de $\pm 1,5 \mu \mathrm{m}$. Les stabilités de la vitesse dans l'eau et de la géométrie des supports ne peuvent être assurées qu'à ce prix.

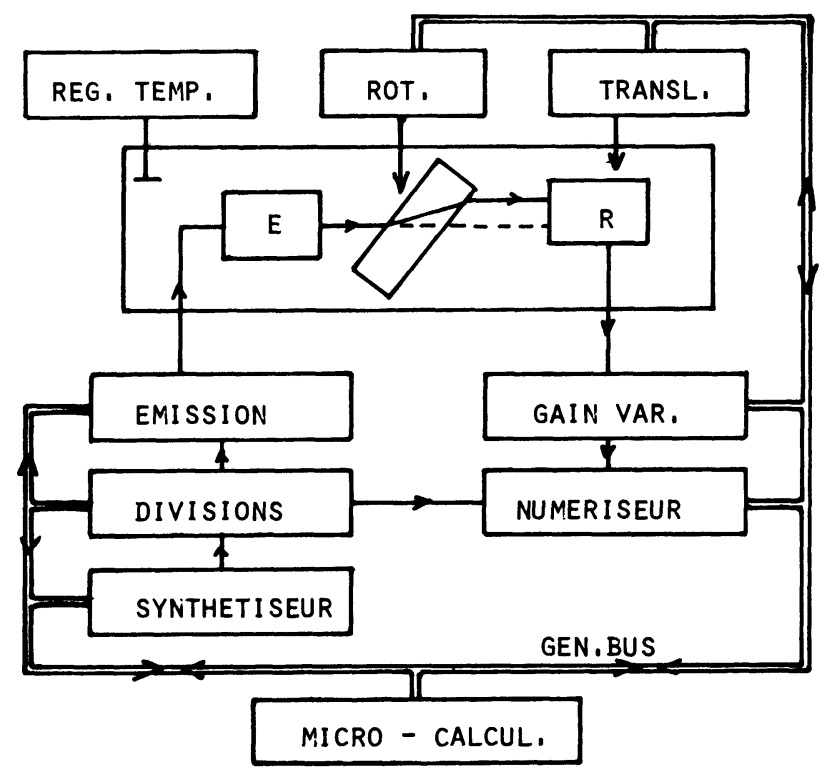

Fig. 2. - Spectro-interféromètre assisté par ordinateur. Synoptique.

[Computer assisted spectro-interferometer.]

La branche de référence n'est alors plus nécessaire, celle-ci pouvant être acquise et mémorisée en début de manipulation par une acquisition sans échantillon. Les fonctions retard et mise en coïncidence étant réalisées par traitement numérique comme on le verra plus loin. La référence et les signaux de mesures sont donc issus de la même paire de transducteurs.

Deux degrés de liberté pilotés par le micro-ordinateur contrôlent l'un, la mise en incidence de l'échantillon, l'autre, en translation perpendiculaire, la poursuite de l'axe du faisceau borné déplacé par la réfraction; cette poursuite étant à l'évidence indispensable pour les mesures d'amplitudes transmises.

La numérisation est précédée d'un étage à gain variable de 0 à $-120 \mathrm{~dB}$ par pas de $1 \mathrm{~dB}$ à commạnde numérique qui permet d'exploiter au mieux la dynamique du convertisseur $\mathrm{A} / \mathrm{N}$ entre l'acquisition de la référence sans échantillon et l'extinction, au voisinage 
d'un angle limite, du mode poursuivi. Les temps de cycle et de retard entre émission et numérisation sont obtenus par division entière d'une fréquence mère issue d'un synthétiseur dont la résolution de 8 digits et la stabilité $<2 \times 10^{-8}$ vont assurer la précision de la chronométrie.

2.2 Mesure Des VITESSES. - Afin d'illustrer simplement les méthodes utilisées, prenons le cas d'une propagation par ondes planes dans un milieu isotrope peu dispersif. Il est bien connu que la propagation d'une onde est un processus causal, linéaire, invariant par translation dans le temps qui peut être décrit par un opérateur de convolution.

Dans le domaine temporel, la réponse $s(t)$ recueillie au récepteur à une excitation $e(t)$ sera :

$$
s(t)=e(t) * h(t)
$$

où $h(t)$ représente la réponse impulsionnelle de l'échantillon dans les conditions de l'expérience, * l'opérateur de convolution temporelle usuel.

$e(t)$ étant connue par l'acquisition du signal de référence en l'absence d'échantillon, $s(t)$ par la mesure avec échantillon, les propriétés du milieu seront décrites par $h(t)$ seront donc classiquement issues d'une opération de déconvolution.

La transformation de Fourier par rapport au temps appliquée à (1) conduit à la relation :

$$
S(v)=E(v) \cdot H(v)
$$

$v$ désignant la variable fréquence du domaine spectral, $H(v)$ la fonction de transfert, transformée de Fourier de la réponse impulsionnelle. Le problème est théoriquement résolu par :

$$
H(v)=\frac{S(v)}{E(v)}=M(v) \exp -i \phi(v) .
$$

La courbe de phase spectrale $\phi(v)$ est riche d'informations sur le comportement mécanique dans la bande de fréquence explorée. Ses écarts à la loi linéaire peuvent montrer des résonances de réseau ou des relaxations structurales. Pour un milieu homogène peu absorbant suivant la loi normale de dispersion dans la bande utile $\pm \Delta v$ d'un transducteur de fréquence centrale $v_{0} ; \Delta v \ll v_{0}$ on pourra écrire :

$$
H(v) \simeq M_{0} \exp (i k(v) e) ; \quad M_{0} \simeq \mathrm{Cte}
$$

$e=$ épaisseur traversée $k(v)=\frac{2 \pi v}{C}=$ nombre d'onde.

La réponse d'un transducteur à une impulsion brève peut se mettre sous la forme :

$$
e(t)=a(t) \cos 2 \pi v_{0} t
$$

fonction à support borné définie pour $t \in(-T / 2$, $+T / 2)$. Sa transformée de Fourier s'écrit alors :

$E(v)=A(v) *\left\{1 / 2 \delta\left(v+v_{0}\right)+1 / 2 \delta\left(v-v_{0}\right)\right\}$ également à support borné pour $v \in\left\{-v_{0}-\Delta v\right.$; $\left.v_{0}+\Delta v\right\}$.

On se trouve donc dans les conditions où l'échantillonnage correct respectant le théorème de Shannon, les algorithmes de transformée rapide de Fourier peuvent être utilisés sans perte d'information et sans résultats fantômes. Le problème de la déconvolution par (3) semble donc apparemment résolu.

On montre aisément [12] qu'en portant (6) et (4) dans (2), la transformée de Fourier inverse de (2) donne :

$$
s(t)=M_{0} a\left(t-\theta_{2}\right) \cos 2 \pi v_{0}\left(t-\theta_{1}\right)
$$

avec

$$
\begin{aligned}
& \theta_{1}=\frac{e}{2 \pi v_{0}} k\left(v_{0}\right) \rightarrow C_{\text {phase }}=e / \theta_{1} \\
& \theta_{2}=\left.\frac{e}{2 \pi} \frac{\partial k}{\partial v}\right|_{v_{0}} \rightarrow C_{\text {groupe }}=e / \theta_{2} .
\end{aligned}
$$

Les deux termes $k\left(v_{0}\right)$ et $\left.\frac{\partial k}{\partial v}\right|_{v_{0}}$ s'obtiennent théoriquement en opérant une régression linéaire sur les points expérimentaux puisque :

$$
\begin{aligned}
k(v) & =\frac{\phi(v)}{e}=\frac{1}{e}\{(\arg E(v)-\arg S(v)\} \\
& =\frac{1}{e}\left\{\operatorname{artg} \frac{Y_{\mathrm{E}}}{X_{\mathrm{E}}} \operatorname{artg} \frac{Y_{\mathrm{R}}}{X_{\mathrm{R}}}\right\}
\end{aligned}
$$

où $X_{\mathrm{E}, \mathrm{S}}$ et $Y_{\mathrm{E}, \mathrm{S}}$ sont les suites représentant les parties réelles et imaginaires des transformées de Fourier discrètes $E(v)$ et $S(v)$. Des difficultés surgissent immédiatement dans l'application concrète de ces relations. En effet, i) les divisions numériques donnent une grande dispersion aux extrémités de la bande passante;

ii) les fonctions artg sont définies modulo $\pi$, et il faut donc rabouter les segments à chaque saut;

iii) enfin et surtout l'ordonnée au centre n'est connue qu'à $2 n \pi$ près, l'ambiguité ne pouvant être levée que par une extrapolation jusqu'à $v=0$ de la courbe de phase. Cette dernière méthode est d'ailleurs la seule utilisable dans le cas des lames à faces parallèles au-delà du premier angle limite pour lesquelles on a pu montrer [13] que le coefficient de transmission complexe contient un terme de phase indépendant de la fréquence.

En conclusion, la précision que l'on peut attendre du traitement dans le domaine fréquentiel seul est médiocre et nous ne l'utilisons que comme appoint dans le traitement du domaine temporel. La mise en évidence d'anisotropies faibles et de la biréfringence acoustique naturelle ou provoquée demande des précisions que nous n'avons pu atteindre que par des méthodes de filtrage-corrélation. D'après (7) et (8) il est clair que dans une bande étroite $\pm \Delta v$ autour de la fréquence centrale $v_{0}$, en dehors d'une résonance de structure ou une relaxation à $v_{0}$, la courbe de 
phase $\phi(v)$ sera un segment de droite passant par l'origine, alors $\theta_{2} \simeq \theta_{1}=\theta$ et $e / \theta$ est une mesure de la vitesse de phase. La propagation est donc équivalente à un retard pur et $s(t)=e(t-\theta)$. L'intercorrélation

$$
C_{\mathrm{es}}(\theta+\delta)=\int_{-\infty}^{+\infty} e(t) e\{t-(\theta+\delta)\} \mathrm{d} \delta
$$

est telle que $C_{\text {es }}(0) \geqslant C_{\text {es }}(\delta)$.

Ainsi le maximum de cette fonction a lieu pour $\delta_{\mathrm{m}}$ tel que $\delta_{\mathrm{m}}+\theta=0$.

L'organigramme de la figure 3 montre les étapes du calcul sachant que les suites d'échantillons vérifient la relation :

$$
C_{\mathrm{ES}}=T F D^{-1}\left\{F(v) T F D^{+1}(E) . T F D^{-1}(S)\right\}
$$

où $T F D^{ \pm 1}$ désignent les transformées de Fourier discrètes directes et inverses; $F(v)$ un filtre numérique adapté au problème traité.

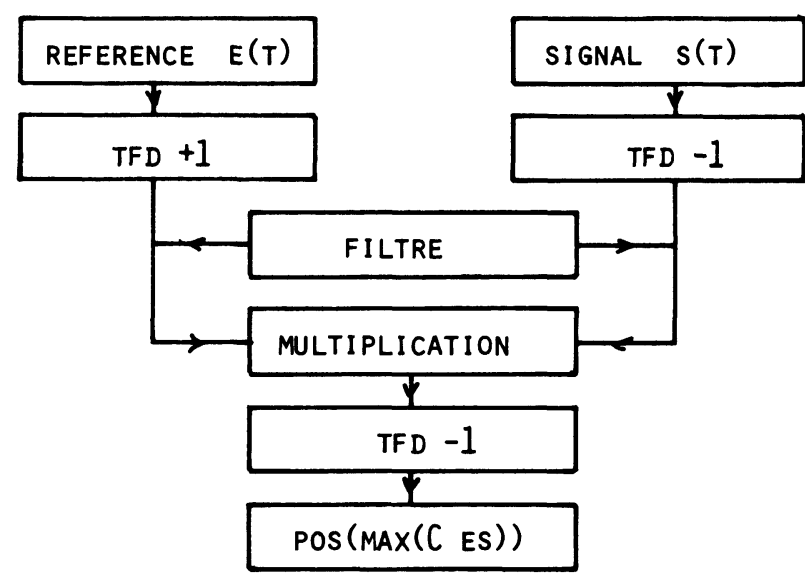

Fig. 3. - Mesure des retards par filtrage-corrélation.

[Correlation filtering method for time delay estimation.]

Si $F(v)$ est un passe-bande étroit centré sur $v_{0}$; pos (max $C_{\mathrm{ES}}$ ) donnera une mesure de $\theta_{1}$ et donc de la vitesse de phase.

Si $F(v)$ est un éliminateur de bande ne conservant que la modulation pos $\left(\max C_{\mathrm{ES}}\right)$ sera une mesure de $\theta_{2}$ donc de la vitesse de groupe. Dans le cas du passebande étroit, le balayage de la bande utile du ou des transducteurs conduit à une spectroscopie de la vitesse de phase en conservant une précision que l'on ne peut obtenir dans le seul domaine spectral.

L'amélioration de la précision s'obtient par deux moyens en remarquant que :

i) La position du $\max C_{\mathrm{ES}}$ n'est connue qu'à $\pm p$, $p$ étant le pas d'échantillonnage temporel. Il ne peut pas être indéfiniment diminué car les registres d'acquisition devant contenir la totalité du signal et le retard maximum voient leurs capacités devenir trop importantes pour les moyens de calcul et d'acquisition. ii) Ce pas ne peut être abaissé au-dessous de la stabilité des horloges d'échantillonnage et des incertitudes de déclenchement. On améliore la précision de la détection de coïncidence au-dessous du pas d'échantillonnage par interpolation linéaire de la dérivée sachant que :

$$
\begin{aligned}
C_{\mathrm{ES}}^{\prime}=T F D^{-1}\left\{i 2 \pi v F(v) . T F D^{+1}\right. & (E) \times \\
& \left.\times T F D^{-1}(S)\right\} .
\end{aligned}
$$

Les erreurs du déclenchement et la non-uniformité d'échantillonnage sont minimales en travaillant toujours autour du retard nul par une translation précise des origines d'acquisition. Cette méthode a l'avantage de laisser immobile la fenêtre temporelle (Hanning) utilisée pour la poursuite des modes. La figure 4 donne la représentation temporelle des signaux échan-
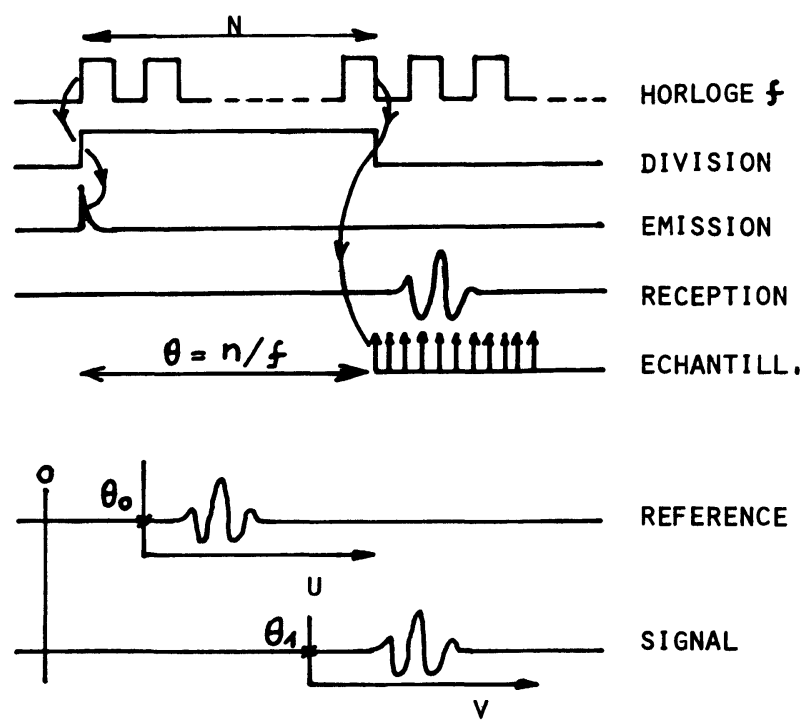

Fig. 4. - Signaux et séquences pour la méthode de zéro.

[Signals and process for sampling starting.]

tillonnés avec des origines différentes obtenues par division entière de la fréquence d'un synthétiseur programmable. Ainsi par translation dans le temps, les fonctions échantillonnées sont :

$$
\begin{aligned}
& e(t) \stackrel{\theta_{a}}{\rightarrow} e(u)=e\left(t-\theta_{0}\right) ; \quad \theta_{0}=n / f_{0} \\
& s(t) \stackrel{\theta_{1}}{\rightarrow} s(v)=s\left(t-\theta_{1}\right) ; \quad \theta_{1}=n / f_{1}
\end{aligned}
$$

$n$ étant l'entier commun de division des fréquences $f_{0}$ et $f_{1}$.

On pourra donc écrire que :

$$
s(v) \simeq M_{0} e\{u-(\theta-\delta)\} ;
$$

$\delta=\theta_{1}-\theta_{0}$ est le retard relatif introduit par ajustement de $f_{1}$ tel que pos $\left(\max C_{\mathrm{ES}}\right)=0 \pm \varepsilon$ affiné par interpolation de $C_{\mathrm{ES}}^{\prime}$, soit $\theta=\delta \pm \varepsilon=\bar{\theta}_{1}-\theta_{0} \pm \varepsilon$ :

$$
\theta=n\left\{1 / f_{1}-1 / f_{0}\right\} \pm \varepsilon
$$


où $f_{1}$ et $f_{0}$ sont connus avec la résolution de 8 digits du synthétiseur de stabilité $<2 \times 10^{-8}$ et $\varepsilon$ l'erreur systématique de détection de coïncidence qui peut être amenée au $1 / 100^{\mathrm{e}} \mathrm{du}$ pas d'échantillonnage.

A titre d'exemple, un mode isolé, de fréquence centrale $1 \mathrm{MHz}$ échantillonné en 128 points au pas de $0,1 \mu$ s soit $12,8 \mu$ s de support total est correctement poursuivi à \pm 1 ns près en trois passages au plus dans la boucle de la figure 5 .

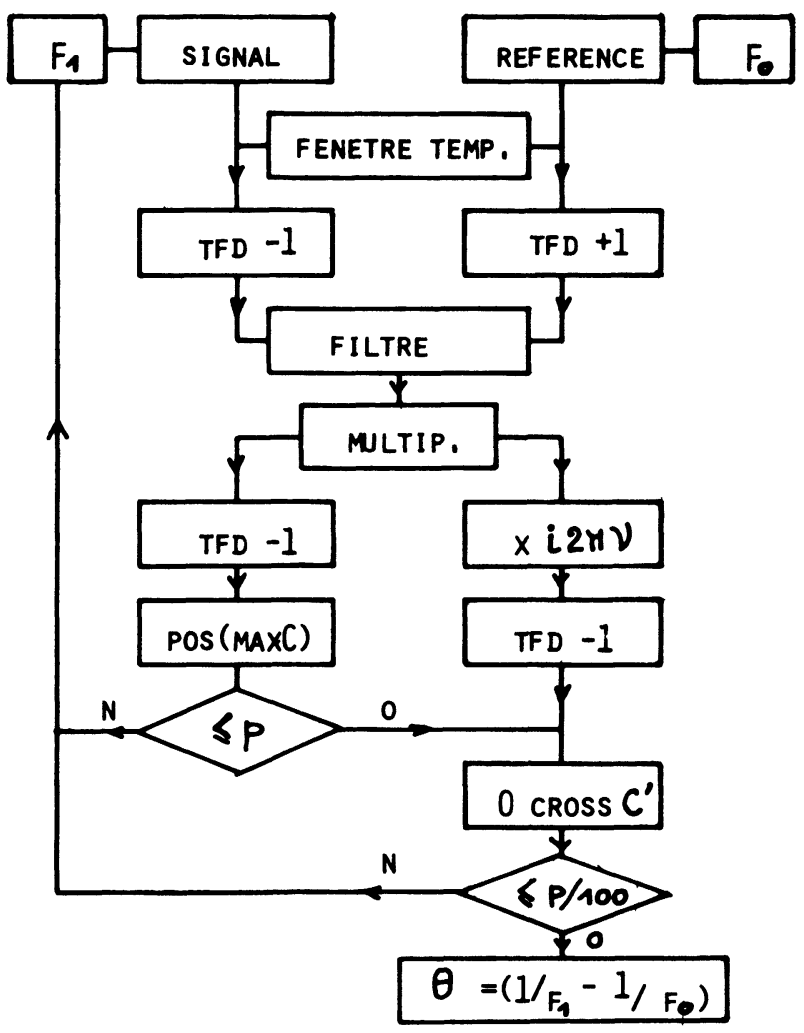

Fig. 5. - Boucle complète avec asservissement d'origine.

[Feedback-loop for zero-crossing and time origin shift.]

Il n'en va plus de même en présence de modes partiellement superposés - cas des épaisseurs faibles et des angles d'incidence faibles - où onde longitudinale et onde transversale interfèrent.

Si l'enveloppe de la fonction de corrélation possède deux maxima séparés par un minimum résolu par la quantification et dont la distance temporelle est inférieure à deux périodes de la fréquence fondamentale, la boucle de poursuite hésite indéfiniment entre ces deux valeurs. On trouvera en [14] la définition d'un critère de séparation, les erreurs associées à la présence de deux modes voisins ainsi que les bases d'un algorithme de séparation de modes pouvant apporter une amélioration sensible dans certains cas.

2.3 Mesures D'AMPLitude. - La mesure de l'atténuation spécifique des matériaux peut être conduite à partir des valeurs crêtes des signaux $e(t)$ et $s(t)$, comme il est d'usage avec les instruments analogiques.
On sait que pour des raisons diversès tenant tant à la nature des matériaux testés, qu'aux faibles performances des détecteurs de crête, on n'aboutit généralement qu'à une évaluation relative des amortissements.

Il est évident d'après l'algorithme de la figure 5 que l'on dispose sans calculs supplémentaires des transformées de Fourier de la référence et du signal de mesure. On accédera donc directement (cf. (3)) à $M_{0}^{2}=\{\overline{M(v)}\}^{2}$ en faisant dans chaque bande de fréquence le rapport des densités spectrales de puissance des deux signaux.

Ainsi, pour chaque incidence, et pour une seule acquisition, parallèlement à la mesure de la vitesse, on pourra calculer le module du coefficient de transmission de la lame à faces parallèles.

\section{Analyse des résultats et caractérisation des maté- riaux.}

Nous laisserons de côté le cas des solides homogènes et isotropes pour lesquels l'identification des paramètres et leur mesure ne pose aucun problème particulier. La caractérisation de composites naturels et artificiels étant l'objet essentiel de nos travaux, nous présenterons quelques cas particulièrement représentatifs. Il est extrêmement rare de n'avoir, avant toute mesure, aucune information sur la texture du matériau, ne serait-ce que par son observation visuelle. On pourra donc situer approximativement quelques classes de symétrie possibles, l'orientation présumée des axes principaux, l'échelle des longueurs d'ondes pour laquelle un modèle de milieu continu est acceptable.

Prenons le cas d'un composite fibres de verrerésine époxy à fibres longues assez bien orientées autour d'un axe principal - axe 3. Dans le plan perpendiculaire $(1,2)$ suivant la distribution géométrique des fibres plusieurs symétries peuvent être rencontrées [15].

a. maille rectangulaire : orthorhombique : 9 constantes $C_{i j}$;

b. maille carrée : quadratique : 6 constantes;

c. maille hexagonale : hexagonal : 5 constantes;

d. distribution aléatoire : unidirectionnel : 5 constantes.

Il est quelquefois difficile de trancher car les matériaux n'ont pas toujours une géométrie bien définie, si bien qu'il peut exister des cas intermédiaires [16]. Nous avons donc réalisé un logiciel d'aide à la décision s'appuyant sur la valeur de l'écart type des mesures de vitesses par rapport au modèle testé et sur une jauge d'anisotropie [17] du type :

$$
\begin{aligned}
& A=2 C_{44} /\left(C_{11}-C_{12}\right) \\
& A^{\prime}=2 C_{66} /\left(C_{11}-C_{12}\right)
\end{aligned}
$$

dont l'écart à l'unité dans les plans d'isotropie per- 
mettra de choisir le modèle optimal ou d'infirmer les hypothèses premières faites sur la texture du matériau [14].

3.1 ACQUiSITION DES DONNÉES. - L'échantillon étant placé dans l'interféromètre, on relève les courbes des lenteurs expérimentales (Figs. 6a, b) et des coefficients de transmission pour les plans $(1,2)$ et $(1,3)$, (Figs. 7a, b), en fonction de la direction de propagation dans le matériau en poursuivant les modes longitudinaux et transverses.

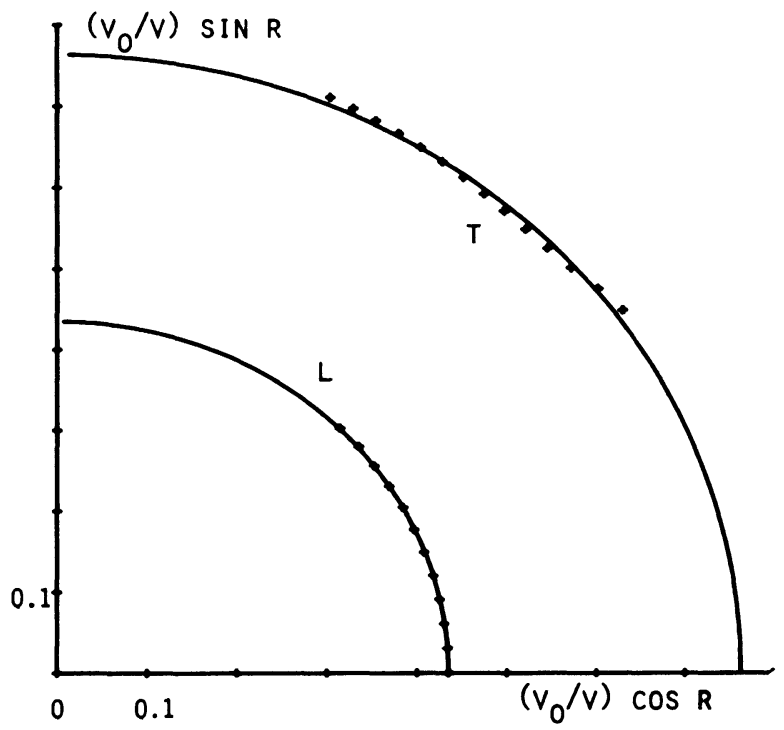

a)

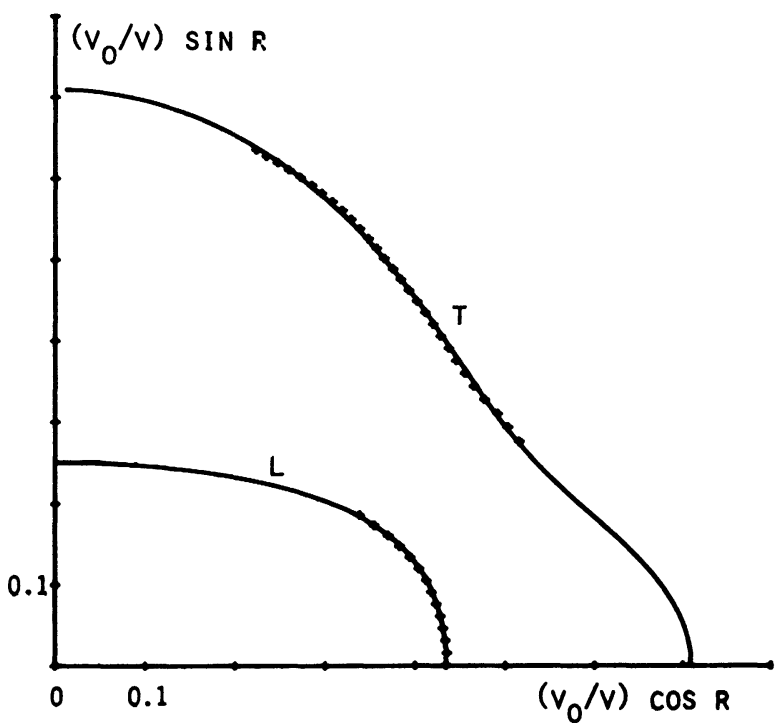

b)

Fig. 6. - Courbes des lenteurs normalisées pour un composite unidirectionnel fibre de verre-résine époxy à $1 \mathrm{MHz}$; a) plan $(1,2)$ d'isotropie transverse, b) plan $(1,3)$ anisotrope.

[Slowness curves for a glass-fibre-epoxy material at $1 \mathrm{MHz}$; a) isotropic plane $(1,2)$, b) anisotropic plane $(1,3)$.]

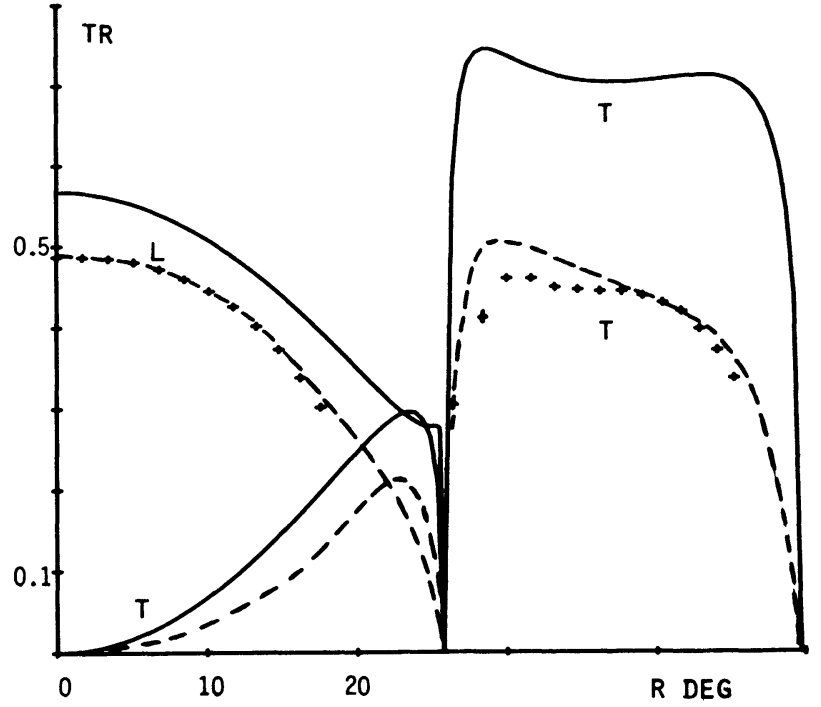

a)

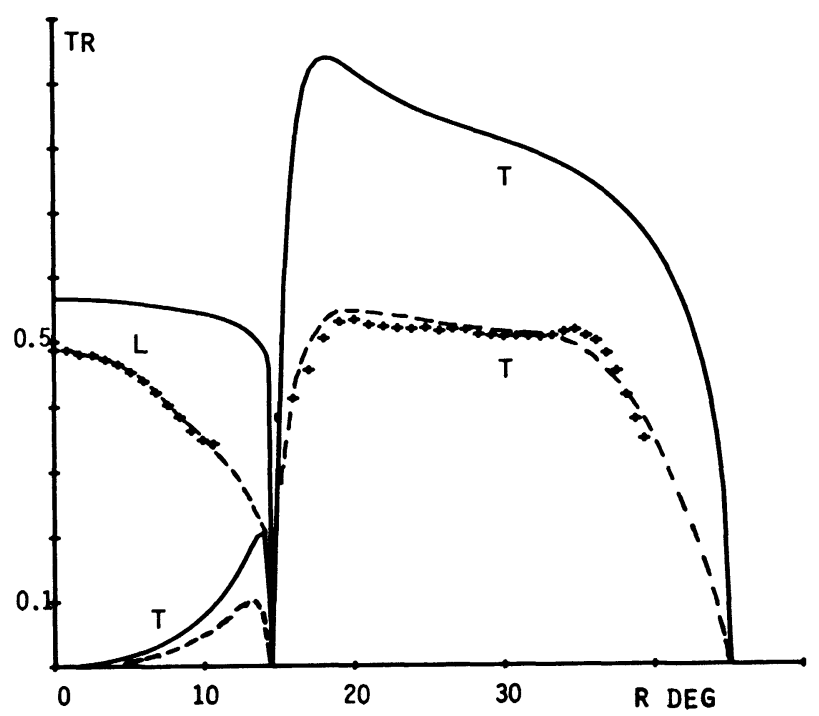

b)

Fig. 7. - Coefficients de transmission en fonction de l'angle d'incidence dans un composite fibre de verre époxy à $1 \mathrm{MHz}$. courbes théoriques du matériau supposé non absorbant, - - - résultat de l'optimisation de $Y=\left\{\eta_{i j}\right\}$; a) plan $(1,2)$ isotrope, b) plan $(1,3)$ anisotrope.

[Transmission coefficients versus incidence angle in a glass-fibre-epoxy plate at $1 \mathrm{MHz}$. - theoretical curves for a lossless assumed medium, - - - - result of $\left(\eta_{i j}\right)$ matrix optimization; a) isotropic plane, b) anisotropic plane.]

3.2 Algorithmes D'OPTIMISATION DES CONSTANTES ÉLASTIQUES. - Le matériau étant $a$ priori réputé homogène à la fréquence utilisée, les modes propagés sont les solutions de l'équation de propagation [17] :

$$
\Gamma_{i l} P_{l}=\rho v^{2} P_{i}
$$

$P_{i}$ étant les composantes du vecteur polarisation de l'onde $\Gamma_{i l}=C_{i j k l} n_{j} n_{k}$ le tenseur de propagation. 
Les vitesses de propagation seront donc les solutions de l'équation caractéristique :

$$
\left|\Gamma_{i l}-\rho v^{2} \delta_{i l}\right|=0 .
$$

Pour un matériau orthotrope ( 9 constantes), $r$ étant l'angle de transmission dans le matériau, pour le plan $(1,2)$ les solutions de (11) s'écrivent :

$$
\begin{gathered}
2 \rho v_{\mathrm{L}, \mathrm{T}}^{2}=A \pm \sqrt{A^{2}-4 B} \\
A=C_{11} \cos ^{2} r+C_{22} \sin ^{2} r+C_{66} \\
B=\left(C_{11} \cos ^{2} r+C_{66} \sin ^{2} r\right)\left(C_{66} \cos ^{2} r+C_{22} \sin ^{2} r\right)- \\
-\left(C_{12}+C_{66}\right)^{2} \cos ^{2} r \sin ^{2} r .
\end{gathered}
$$

$\mathbf{L}$ et $\mathbf{T}$ désignant respectivement les modes quasi longitudinal et quasi transverse.

$C_{11}$ est connue au départ par une mesure en incidence normale. Les trois inconnues $C_{22}=X_{1}$; $C_{66}=X_{2} ; C_{12}=X_{3}$ fournissent les composantes du vecteur à optimiser pour toutes les valeurs expérimentales de $v^{2}$ déduites des courbes de lenteur [18]. Les deux modes sont traités simultanément en élevant (12) au carré car le vecteur optimal $X$ doit être évidemment le même pour tous les modes de chaque plan.

On obtient ainsi une fonctionelle du type :

$$
F(X)=\sum_{k=1}^{N}\left\{f_{k}(X)\right\}^{2}
$$

\section{$N=$ nombre de données expérimentales}

que l'on minimise par une méthode itérative de type Newton mettant en ouvre le gradient et la matrice Hessienne de la fonctionnelle. On trouvera en [14] l'exposé détaillé des problèmes de convergence et les calculs de variance conduisant à l'estimation des erreurs sur $X$.

Les courbes en trait plein des figures $6 \mathrm{a}$ et $6 \mathrm{~b}$ sont les lenteurs recalculées à partir de la valeur optimale de $X$ déduite des points expérimentaux pour le composite fibre de verre-résine époxy.

A titre d'exemple, les données expérimentales recueillies à $1 \mathrm{MHz}$ dans le plan $(1,2)$ perpendiculaire aux fibres, donnent après optimisation :

$C_{11}=25,01 \pm 0,8 \% ; \quad C_{66}=7,87 \pm 1,3 \%$;

$C_{12}=9,00 \pm 2 \%($ en GPa $)$;

$\sigma_{\mathrm{L}}=0,15 \%: \sigma_{\mathrm{T}}=0,76 \%$ écarts types sur les vitesses $V_{\mathrm{L}}$ et $V_{\mathrm{T}}$

$A^{\prime}=2 C_{66} / C_{11}-C_{12}=0,98$.

La jauge d'anisotropie voisine de 1 et la faiblesse des écarts types valident donc le modèle hexagonal à $1 \mathrm{MHz}$. La spectroscopie de ce matériau de 1 à $5 \mathrm{MHz}$ montre [14] une dispersion croissante avec la fréquence et un écart croissant avec le modèle de symétrie hexagonale.
On trouvera toujours en [14] d'autres exemples montrant des identifications de structures pour un composite titane-carbure de silicium ou des composites naturels tels que le bois et un schiste ardoisier pour lesquels des modèles ont pu être établis et validés avec les intervalles de confiance correspondants.

3. 3 Evaluation Des amORTISSEMENTS INTERNES. La connaissance des $C_{i j}$ permet le calcul des coefficients de transmission [13] de la lame en matériau supposé non absorbant. Les courbes en traits pleins des figures $7 \mathrm{a}$ et $7 \mathrm{~b}$ montrent ces résultats. Compte tenu de l'absorption, les points expérimentaux sont évidemment en dessous.

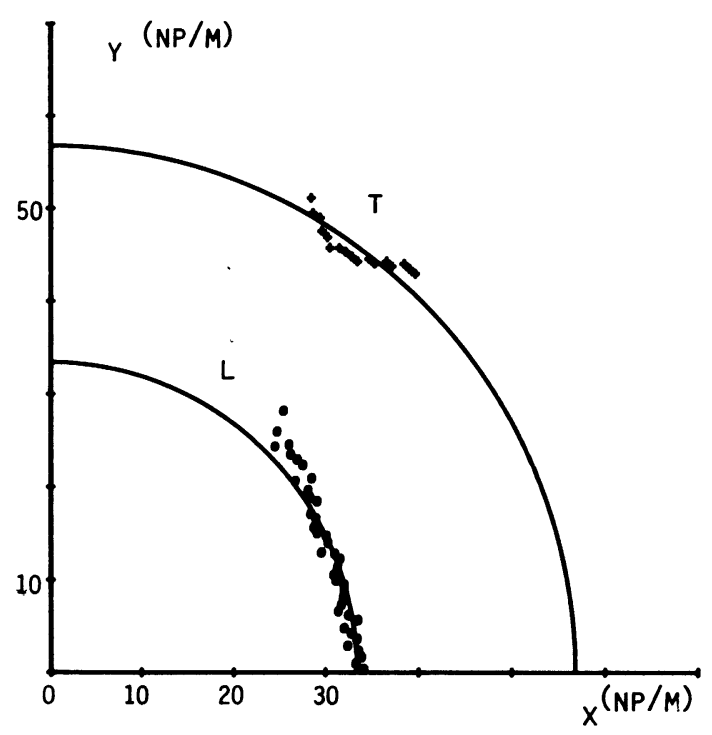

a)

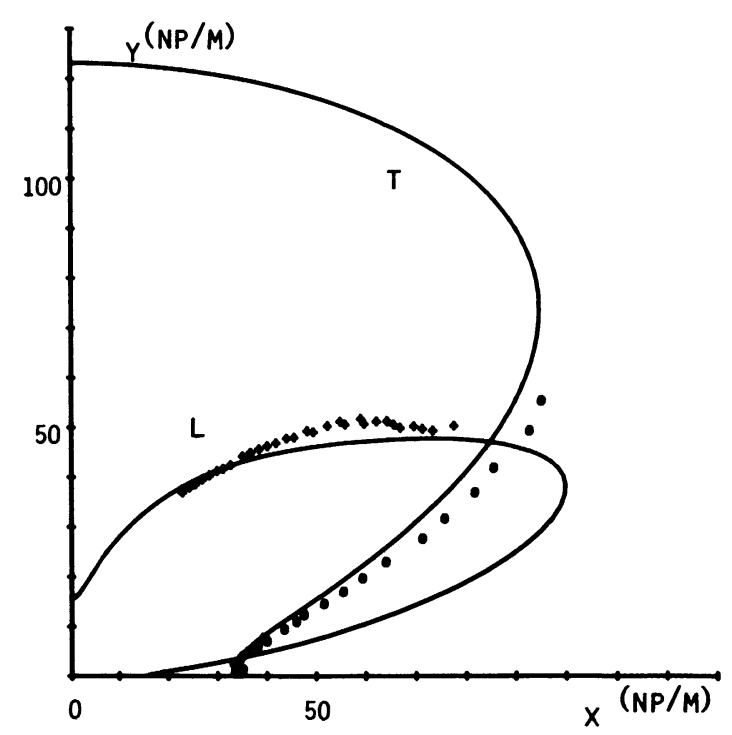

b)

Fig. 8. - Partie imaginaire du nombre d'onde en fonction de la direction de propagation; a) plan $(1,2)$ isotrope, b) plan $(1,3)$ anisotrope.

[Imaginary part of wave number versus propagation angle; a) isotropic plane, b) anisotropic plane.] 
Dans une bande fréquence assez étroite centrée sur $v_{0}$, on pourra toujours reconsidérer une loi de comportement du type :

$$
C_{i j}^{\prime}=C_{i j}+2 \pi v \eta_{i j}
$$

et tenter par une méthode analogue à celle du paragraphe 3.2 précédent d'optimiser le nouveau vecteur $Y=\left\{\eta_{i j}\right\}$ recalculant au mieux les courbes de transmission calculées sur les points expérimentaux [19]. Nos travaux en cours portent sur la recherche d'une fonctionnelle optimisant ces coefficients de viscosité anisotrope avec une fiabilité et un éventail de possibilités identiques à celles obtenues pour les parties réelles $C_{i j}$. Les courbes en pointillés des figures $7 \mathrm{a}$ et $7 \mathrm{~b}$ montrent le résultat des premiers essais, tandis que sur les figures $8 \mathrm{a}$ et $8 \mathrm{~b}$ on a représenté la partie imaginaire de nombre d'onde en fonction de la direction de propagation.

La dispersion reste relativement importante nécessitant la poursuite d'efforts aussi bien dans le domaine de la dynamique d'acquisition que dans la mise au point d'algorithmes plus performants.

\section{Conclusion.}

La caractérisation structurale et mécanique des solides naturels et surtout de synthèse, tels que les matériaux composites et les céramiques revêt actuellement une grande importance économique.
Toute une classe de problèmes reliant le comportement global aux microstructures demande une connaissance étendue et précise des paramètres mécaniques. Leur évolution en fonction des fréquences (spectroscopie) et en fonction de paramètres extérieurs (contraintes externes et/ou endommagement) peut seule permettre une connaissance approfondie des matériaux.

Les appareillages et techniques que nous venons de présenter ont été conçus dans ce but. La simplicité de la mise en ouvre et la rapidité d'obtention de résultats précis et confirmés en font un instrument capable d'aborder raisonnablement les études statistiques conduites sur des lots d'échantillons représentatifs.

Son utilisation dans le cas des matériaux échappant aux hypothèses de départ nous a déjà révélé d'autres possibilités d'analyse. Des modes guidés et certains couplages fibre-matrice ont ainsi pu être mis en évidence dans des composites $3 \mathrm{D}$ à structure périodique.

Citons enfin, sur un plan plus fondamental, la recherche d'une meilleure précision dans les mesures d'amplitude. La dispersion des résultats d'atténuation dans des matériaux homogènes et isotropes bien connus par ailleurs, nous a conduit à l'étude de la réfraction d'un faisceau borné au travers d'une lame à faces parallèles [20]; on a pu mettre en évidence la perte de symétrie d'un faisceau gaussien d'ondes longitudinales expliquant ainsi l'écart de position à la loi de Descartes que l'on observe expérimentalement.

\section{Bibliographie}

[1] Firestone, F. A., J. Acoust. Soc. Am. 18 (1946) 200.

[2] Holbrook, R. D., J. Acoust. Soc. Am. 20 (1948) 590.

[3] Williams, Lamb, J. Acoust. Soc. Am. 30 (1958) 308.

[4] McSkimin, Andreatch Jr, J. Acoust. Soc. Am. 41 (1967) 1052 et ibid. 34 (1962) 609.

[5] May Jr, J. E., Ire Nat. Conv. Rec. 6 (1958) 134.

[6] Papadakis, E. P., J. Acoust. Soc. Am. 42 (1967) 1045 et J. Appl. Phys. 35 (1964) 1474.

[7] Perviskin, I. I. et Filipov, L. P., Soc. Phys. Acoust. 7 (1962) 307.

[8] Chase, C. E., Phys. Fluids 1 (1958) 193.

[9] Williamson, R. C., Eden, D., J. Acoust. Soc. Am. 47 (1970) 1278.

[10] Markam, M. F., Composites 145 (March 1970).

[11] Smith, R. E., J. Appl. Phys. 43 (1972).

[12] RoDdIER, F., Distributions et transformation de Fourier (Mc Graw-Hill) 1978, ISBN-2-7042-1004-7.
[13] Hosten, B. et Deschamps, M., C. R. Hebd. Séan. Acad. Sc. Paris 299 (1984) $\mathrm{n}^{\circ} 9$.

[14] Castagnede, B., Thèse spécialité, 2001, Univ. Bordeaux I (1984).

[15] Ross, C. A. et Sierakowski, R. L., Shock Vib. Dig. 7 (1975) 96.

[16] Ledbetter, H. M., J. Appl. Phys. 48 (1977) 1874.

[17] Auld, B. A., Acoustic Fields and Waves in solids, vol. 1 (J. Wiley and Sons, N. Y.) 1973.

[18] Hosten, B., Castagnede, B., C. R. Hebd. Séan. Acad. Sc. Paris 296 (1983) 297.

[19] Hosten, B., Deschamps, M., 18e Colloque G.F.R. Paris (nov.-déc. 1983).

[20] Hosten, B., Deschamps, M., C. R. Hebd. Séan. Acad. Sc. Paris 299 (1984) no 15. 